\title{
MeDIP-Seq and RNA-Seq Releaved TGFB2 Associated with Disease Resistance to ALV-J Infection in Chickens
}

\section{Yiming Yan}

South China Agricultural University

Huihua Zhang

Foshan University

Shuang Gao

South China Agricultural University

Huanmin Zhang

USDA-ARS EAST Lansing

\section{Xinheng Zhang}

South China Agricultural University

\section{Weiguo Chen}

South China Agricultural University

Wencheng Lin

South China Agricultural University

Qingmei Xie ( $\square$ qmx@scau.edu.cn )

South China Agricultural University

\section{Research}

Keywords: MeDIP-Seq, RNA-Seq, ALV-J-resistant chicken, integrated analysis, TGFB2

Posted Date: December 29th, 2020

DOl: https://doi.org/10.21203/rs.3.rs-135681/v1

License: (1) (1) This work is licensed under a Creative Commons Attribution 4.0 International License. Read Full License 


\title{
MeDIP-Seq and RNA-Seq revealed TGFB2 associated with disease resistance to ALV-J infection in chickens
}

\author{
Yiming Yan ${ }^{1,2,3}$, Huihua Zhang ${ }^{4}$, Shuang Gao ${ }^{1,2,3}$, Huanmin Zhang ${ }^{5}$, Xinheng \\ Zhang $^{1,2,3}$, Weiguo Chen ${ }^{1,2,3}$, Wencheng Lin ${ }^{1,2,3}$, Qingmei Xie ${ }^{1,2,3 *}$
}

1 College of Animal Science, South China Agricultural University and Guangdong Provincial Key Lab of AgroAnimal Genomics and Molecular Breeding, Guangzhou, People's Republic of China

2 Key Laboratory of Animal Health Aquaculture and Environmental Control, Guangdong, Guangzhou, People's Republic of China

3 South China Collaborative Innovation Center for Poultry Disease Control and Product Safety, Guangzhou, People's Republic of China

4 College of Life Science and Engineering, Foshan University, China

5 USDA, Agriculture Research Service, Avian Disease and Oncology Laboratory, East Lansing, Michigan, USA

\section{* Correspondence:}

Qingmei Xie

qmx@scau.edu.cn

Keywords: MeDIP-Seq, RNA-Seq, ALV-J-resistant chicken, integrated analysis, TGFB2. 


\section{Abstract}

\section{Background}

Avian leukosis virus subgroup J (ALV-J) is an oncogenic virus that causes serious economic losses in the poultry industry; unfortunately, there is no effective vaccine. Therefore, ALV-J-susceptible and -resistant chickens had been screened in this study. DNA methylation plays a crucial role in several biological processes, and an increasing number of diseases were proven to be related to alterations of DNA methylation. Thus, we generated and provide the genome wide genome-expression and DNA-methylation profiles by MeDIP-Seq and RNA-Seq in ALV-J-susceptible and -resistant chickens.

\section{Results}

8304 DMRs were identified by MeDIP-Seq analyses and 515 differentially expressed genes were identified by RNA-Seq analysis. Through the results of integration analysis, we screened six candidate genes for screening ALV-J-resistant chickens that were aberrant methylation in the promoter region. Furthermore, functional analyses showed that overexpression of TGFB2 promoted ALV-J replication, which suggested TGFB2 played an important role in disease resistance to ALV-J infection in chickens.

\section{Conclusions}

The present study provides new insights into understanding epigenetic changes in ALV-J-susceptible and -resistant chickens, and results provide a basis for breeding disease-resistant-chicken lines. 


\section{Background}

As a member of the Alpharetrovirus genus, avian leukosis virus (ALV) causes different pathotypes of neoplastic disease in chickens [1,2]. The spread of ALV causes slow growth and production-performance degradation to the chicken industry [3]. According to host range and viral-envelope protein, ALVs can be classified into ALVA, -B, -C, -D, -E, -J, and -K subgroups [4,5]. ALV-J was first isolated from meat-type chickens in England in 1988 [4]. Since then, ALV-J has been the most prevalent subtype of ALVs [6,7]. Till now, there is no effective vaccine against ALV-J. The eradication managements is an effective control measure to control this disease $[8,9,10,11]$

DNA methylation, a major epigenetic mechanism, plays a crucial role in several biological processes, such as the regulation of gene expression, embryonic development, X-chromosome inactivation, and oncogenesis $[12,13,14,15,16]$. DNA methylation maintains a stable state without stimulating the environment, but many factors also can change DNA-methylation patterns in organisms, including senility, development, and diet [17]. Due to the potential influence on complex traits, series of diseases proved to be related to the alterations of DNA methylation, such as psychosis, diabetes, and many kinds of cancer $[18,19]$. Although the relationship between DNA methylation and gene expression is still unclear, the methylation of the gene-promoter region cuases the transcriptional repression [20]. A number of studies indicated that many tumorsuppressor genes, such as FHIT, PTEN, and CMTM3, are silenced by promoter hypermethylation in the development of lung, gynecological, and gastric cancer $[21,22,23]$. The epigenetic mechanisms responsible for disease resistance in chicken is still poorly understood.

Infectious diseases are major challenges for the poultry industry that causes widespread production losses. Presvisous reports indicated that different chicken lines present sgnifacantly different levels of resistance to certain diseases or immunogens, such as Marek's disease [24], avian influenza [25], Escherichia coli [26] and Campylobacter [27]. In our previous study, we assessed chicken's genetically resistance 
to ALV-J. A total of eight Chinese broiler lines were employed to infect ALV-J, the line which has the lowest infection proportion was deemed to low susceptibility to ALV-J. Then, the cocks and hens of this chicken line were challenge with ALV-J, and the resistant chickens was screened as described in Materials and Methods. Using ALVJ-resistant chickens, we identified series of tumor-related genes and oncogenous circular RNA (circRNA) [28]. However, few reports regarding the impact of DNA methylation on ALV-J-resistance in birds. This study focused on the difference of DNA methylation and gene expression between ALV-J-resistant and -susceptible chickens. In this study, we performed MeDIP-Seq and RNA-Seq to identify the differences of DNA methylation between ALV-J-resistant and -susceptible chickens. Our study provides the foundations for research into the mechanisms behind the pathogenicity, and a basis for screening ALV-J-resistant chickens.

\section{Methods}

\section{Ethics Statement}

The animal study protocol was approved by the South China Agricultural University Committee of Animal Experiments (approval ID SYXK-2014-0136). Experiments were closely followed in accordance with the recommendations of the Guide for the Care and Use of Laboratory Animals of the National Institutes of Health.

\section{Resistance of different chicken lines to ALV-J infection}

In this wok, eight Chinese broiler lines were used (100 chickens per line). Chickens were intraperitoneally inoculated ALV-J NX0101 at a dose of $0.2 \mathrm{~mL}\left(10^{4.5}\right.$ $\mathrm{TCID}_{50} / 0.1 \mathrm{~mL}$ ) at one-day-old, and inoculated once again at 5 days old. RT-PCR detection, virus isolation, antibody detection and ALV-P27 antigen capture ELISA were used to detect the infection of ALV-J. The positive infection rate of each chicken line was calculated to identify the most resistant line to ALV-J infection.

Screening of ALV-J-resistant chickens 
The chicken line with the lowest infection proportion continued to be used in this work. We chose the chickens which were not infected were challenged with ALV-J for controlled mating, and the newly hatched chickens (F1 generation) were intraperitoneally inoculated ALV-J NX0101 at one-day-old and at 5 days old. Whole blood and anticoagulant blood post-challenge was collected for detection using reverse transcription PCR (RT-PCR), virus isolation, P27 group specific antigen detection by ELISA, and antibody detection. Twenty weeks later, the chickens that tested without infection with ALV-J from beginning to end were identified as ALV-J-resistant chickens, and the others were identified as ALV-J-susceptible chickens. Each generation of ALV-J-susceptible chickens were removed and ALV-J-resistant chickens used for controlled mating. The newly hatched chickens were screened as mentioned above. The chickens were raised in negatively pressured biosecurity isolators under quarantine conditions, and provided with water and feed ad libitum.

\section{Sample}

Three ALV-J-resistant chickens and three ALV-J-susceptible chickens from F4 generation had been chose, and the livers of them were rapidly collected, immediately snap-frozen using liquid nitrogen, and stored at $-80{ }^{\circ} \mathrm{C}$. The livers were detected by IFA and collected for MeDIP-Seq and RNA-Seq. After this study, the remaining chickens were released to the population for breed conservation.

\section{MeDIP-Seq}

Genomic DNA from samples were isolated using phenol-chloroform, precipitated with ethanol, and sonicated to 100-500 bp using Bioruptor (Diagenode). Sonicated DNA was end-repaired, A-tailed, and ligated to adapters by using NEBNext ${ }^{\circledR}$ Ultra $^{\mathrm{TM}}$ DNA Library Prep Kit (NEB). Then, MeDIP was performed with a monoclonal antibody against 5-methylcytosine by following the manufacturer's standard protocol (Active Motif). MeDIP DNA libraries were quantified using Quant-iT PicoGreen dsDNA Kits (Life Technologies), and subjected to high-throughput 150 base paired- 
end sequencing on Illumina Hiseq sequencer according to the manufacturer's recommended protocol(Cloud-seq Inc., Shanghai, China).

\section{RNA-Seq}

First, total RNA ( $1 \mu \mathrm{g})$ was used for removing rRNAs using Ribo-Zero rRNA Removal Kits (Illumina, San Diego, CA, USA) following the manufacturer's instructions. Second, RNA libraries were constructed by using rRNA-depleted RNAs with TruSeq Stranded Total RNA Library Prep Kit (Illumina, San Diego, CA, USA) according to the manufacturer's instructions. Libraries were controlled for quality, and quantified using the BioAnalyzer 2100 system (Agilent Technologies, Inc., USA). Lastly, 10 pM libraries were denatured as single-stranded DNA molecules, captured on Illumina flow cells, amplified in situ as clusters, and sequenced for 150 cycles on Illumina HiSeq Sequencer according to the manufacturer's instructions(Cloud-seq Inc., Shanghai, China).

\section{Identification of Differentially Methylated Regions}

High-quality MeDIP-seq reads were aligned against the Gallus gallus genome (UCSC galGal4) using Bowtie 2 software (v2.2.4)[29]. Only uniquely mapped reads were used for further analysis. Peak calling was performed with MACS software (v1.4.3)[30]. Differentially methylated regions (DMRs) were identified by diffReps software (v1.55.4)[31]. The p-value of the filtering standard was 0.05, and a 2-fold change in the difference of read numbers was used as a criterion for the DMRs.

\section{Identification of Differentially Expressed Genes}

RNA-Seq high-throughput sequencing and subsequent bioinformatics analysis were done by Cloud-Seq Biotech (Shanghai, China). Briefly, paired-end reads were harvested from the Illumina HiSeq 4000 sequencer, and quality-controlled by Q30. After 3' adaptor-trimming and low quality reads removing by cutadapt software (v1.9.3), the high quality clean reads were aligned to the reference genome (UCSC 
galGal4) with hisat2 software (v2.0.4). Then, guided by the Ensembl gtf gene annotation file, cuffdiff software [32] was used to get the gene level FPKM as the expression profiles of mRNA, and fold change and p-value were calculated based on FPKM, differentially expressed mRNA (DEGs) were identified. Genes with a p value $<0.05$ and a $\log 2$-transformed value smaller than -1 or greater than 1 were considered to be statistically significant DEGs.

\section{MeDIP-Seq Data Validation by Bisulfite Sequencing}

To verify differentially methylated genes between ALV-J-resistant and susceptible chickens, 1 ug of genomic DNA from ALV-J-resistant and -susceptiblechicken samples was treated with sodium bisulfite using the EpiTect Fast LyseAll Bisulfite Kit (QIAGEN, German). Primer sequences for the genes selected for validation are documented in Table S9.

\section{Validation of RNA-Seq Data by Quantitative RT-PCR}

A total of $500 \mathrm{ng}$ RNA was used to synthesize cDNA using PrimeScript RT Reagent Kit (Perfect Real Time, TaKaRa, Osaka, Japan). Quantitative real-time PCR was performed using the 2x SYBR Green qPCR Master Mix (Bimake, USA). PCR conditions were $95^{\circ} \mathrm{C}$ for $5 \mathrm{~min}$, followed by 40 cycles of $95^{\circ} \mathrm{C}$ for 10 seconds, ending at $60{ }^{\circ} \mathrm{C}$ for 30 seconds. Expression levels of each target gene were normalized to the endogenous beta-actin level. Relative gene expression was analyzed using the $2^{-\Delta \Delta \mathrm{Ct}}$ method [33]. Primer sequences for the target genes selected for validation are documented in Table S10.

\section{Integrated Analysis of DMGs and DEGs}

MeDIP-Seq and RNA-Seq data were interpreted in an integrated way to identify epigenetically regulated genes. DEGs that retained DMRs in the regulatory areas were selected as candidate genes. Fold change $>2$ and $p$-value $<0.05$ were used as filtering criteria. All analyses were based on galGal4. 


\section{Gene-Ontology (GO) Annotation and the Kyoto Encyclopedia of Genes and Genomes (KEGG) Pathway}

To further investigate the biological processes and functions associated with differentially expressed genes, we performed gene-ontology (GO) and KEGG pathway analysis. Genes exhibiting more than twofold change in different samples were analyzed for GO enrichments using clusterProfiler [34,35], and KEGG pathway enrichments using the DAVID functional annotation tool (http://david.abcc.ncifcrf.gov/).

\section{Western blotting}

DF-1 cells were subjected to western blot analysis as previously described by Zhang et al [28]. Briefly, the primary antibodies used were polyclonal rabbit antiTGFB2 (1:1000; predicted molecular weight: $48 \mathrm{kDa}$; Absin, China) and $\beta$-actin (1:1000; predicted molecular weight: $42 \mathrm{kDa}$; Bioss Inc.), which was used as a proteinloading control. The secondary antibody was goat polyclonal anti rabbit $\operatorname{IgG}(\mathrm{H}+\mathrm{L})-$ horseradish peroxidase (HRP; Bioss Inc).

\section{Statistical Analysis}

Data were processed using GraphPad Prism (version5.0) and are expressed as the mean $\pm \mathrm{SE}$. The Student's t-test was used to assess differences among groups, where $\mathrm{P}$ $<0.05$ and $\mathrm{P}<0.01$, were considered to show significant differences between the groups. Western blot bands were quantified with Image-Pro Plus 6.0.

\section{Results}

\section{Identification of ALV-J-Resistant and -Susceptible Chickens}

To screen the ALV-J-Resistant and -Susceptible chickens, chickens from F4 generation were intraperitoneally inoculated ALV-J NX0101. RT-PCR detection, virus 
isolation, antibody detection and ALV-P27 antigen capture ELISA had been done every two weeks. Twenty weeks later, we found 10 chickens were tested negative for ALV-J all the time, and 16 chickens were infected by ALV-J (Table S1). Compared to ALVJ-resistant chickens, most of ALV-J-susceptible-chickens showed gradual emaciation. Spleens of ALV-J-susceptible-chickens were significantly bigger than the ALV-Jresistant chickens at 20 weeks (Figure 1A). After incubated with liver homogenate into DF-1 cells, the livers of ALV-J-susceptible-chickens were determined to be positive for p27(Figure 1B). The results showed we got the ALV-J-resistant chickens which had resistance to ALV-J.

\section{Methylomic Profiling of ALV-J-Resistant and Three ALV-J-Susceptible}

\section{Chickens}

To screen the methylomic Profiling of ALV-J-Resistant and Three ALV-JSusceptible Chickens, the livers of them were sampled, and MeDIP-Seq dad been down. As a result, the numbers of raw reads from the three live samples from ALV-J-resistant chickens were 17108 942, 17719 218, and 17343 579, and we obtained 16993 746, 17977 748, and 18087379 raw reads from three ALV-J-susceptible-chicken samples. After the removal of adapter sequences, contamination, and low-quality reads from the raw reads, we obtained 17101 214, 17708 194, 17329 183, 16982 842, 17965 218, and 18075056 clean reads, respectively. Reads were mapped to the reference genome (UCSC galGal4), and mapping rates of $82.13 \%-83.98 \%$ were obtained (Table 1). MeDIP-seq reads were detected in all chromosomal regions (Figure S1).

High methylation peaks were observed in the methylcytosine enrichment region that were key parameters for analyzing DNA-methylation profiles. We analyzed the distribution of peaks across different genomic regions in each sample. Intergenic regions showed more peaks than other regions did. We confirmed the frequency of peaks in each region, including the promoter at the transcription start site, exon, intron, and upstream. DNA methylation in promoter regions is crucial for gene-expression 
regulation and a peak range of $5.11 \%-5.37 \%$ belonged to the promoter regions (Figure 2).

\section{Characterization of Differential Methylated Regions and Validation of} MeDIP-Seq Data Using Bisulfite Sequencing

A differential methylation region (DMR) is regarded as a functional region involved in the regulation of gene-transcription level. DiffReps was used to analyze DMRs in ALV-J-resistant and -susceptible chicken samples. In total, we identified 8304 DMRs; $56.7 \%$ of them were hypermethylated, and $43.3 \%$ were hypomethylated relative to ALV-J-resistant chickens (Table S2). DMR distribution showed that uniquely mapped reads in intergenic regions had a relatively higher methylation level than that of others (Figure 3). To reveal the regulatory mechanisms of disease resistance from a DNA-methylation standpoint, DMRs between groups would be there if a thorough inquiry were made.

To validate MeDIP-Seq data, we mainly focused on DMRs located at the promoter region, which may contribute the regulation of gene expression, so we detected the methylation level of TGFB2 gene. BSP results confirmed data accuracy from MeDIPSeq analysis (Figure 4).

In order to further understand the function of 8304 DMRs, GO analysis was carried out using DAVID software. Those with p-value $<0.05$ were considered as significantly enriched GO. GO analysis showed that 403 terms were enriched, including the positive regulation of transcription from RNA polymerase II promoter, transcription, DNAtemplated, and negative regulation of cell proliferation (Table S3 and Figure 5). Four pathways were also identified $(\mathrm{p}<0.05$, Table $\mathrm{S} 4)$ : steroid biosynthesis, autophagy regulation, beta-alanine metabolism, and $\mathrm{ABC}$ transporters.

\section{Mapping of RNA-Seq Library Sequencing Data}

To identify mRNAs involved in disease resistance, total RNAs from ALV-Jresistant and -susceptible-chicken samples were used to construct small RNA libraries. We obtained 31752 754, 28323 384, and 22442240 clean reads from the ALV-J- 
resistant-chicken library, and 27209 832, 19975 018, and 32350232 clean reads from the ALV-J-susceptible-chicken library (Table 2) after deleting some contaminant reads. In total, 515 significantly differentially expressed mRNAs were identified in ALV-Jresistant chickens compared to in ALV-J-susceptible chickens (Table S5). A heat map of the 515 differentially expressed mRNAs was generated to illustrate the distinguishable mRNA expression profiling of the samples (Figure 6A). Of them, 189 mRNAs were upregulated, and 326 mRNAs were downregulated in ALV-J-susceptible chickens compared to in ALV-J-resistant chickens. To study the biological functions of these mRNAs, GO analysis was performed on differentially expressed genes with $p$ -

value $<0.05$. Significant GO terms showed that these DEGs were related to many biological processes and molecular functions (Table S6). Most of these terms were closely related to the establishment of localization and the cellular development process (Figure 6B). KEGG pathways analysis showed that these DEGs were significantly involved in the glycosphingolipid biosynthesis-globo series, steroid-biosynthesis, and fructose- and mannose-metabolism pathways (Figure 6C and Table S7). Furthermore, qRT-PCR was performed to validate the results of RNA-Seq. In total, qRT-PCR data for 6 mRNAs were consistent with the observed tendencies using RNA-Seq (Figure 6D).

\section{Association Analysis of MeDIP-Seq and RNA-Seq}

Association analysis between methylation and transcriptome was based on MeDIP-Seq and RNA-Seq sequencing data (Table S8). The comprehensive distribution patterns of the genes with both differential methylation and expression levels are illustrated in Figure 7A. After merging overlapping DMGs containing DMRs with different gene elements into the unitary DMG, a total of 3197 DMGs, including 1198 simultaneously hypermethylated and hypomethylated DMGs, were identified. Bidirectional gene-expression patterns were located in various genomic regions (Figure $7 B \& C)$. Furthermore, we observed 72 DEGs that might be regulated by aberrant DNA methylation, including 44 hypermethylated genes showing simultaneous down- 
expression, and 28 hypomethylated genes showing concurrent up-expression, which were considered as potential candidate genes. In particular, TMEM104, FAM173A, CNP1, HSD17B7, SUPV3L1, TGFB2 exhibited differential methylation in the promoter region (Table 3).

Bisulfite Sequencing and qPCR analysis of TGF $\beta 2$ expression level in randomly selected chickens

In order to confirm the results of TGFB2 was hypermethylated in ALV-J-resistant chickens, we analyzed its DNA methylation level in another four random selected ALV-J-resistant and four ALV-J-susceptible chickens from F4 generation. As a result, compare with ALV-J-susceptible chickens, TGFB2 had higher methylation levels, essentially the same as those results revealed by MeDIP-seq(Figure 8A). At the same time, we identified whether TGFB2 was commonly down-expression in ALV-Jresistant chickens, the chickens which mentioned above had been analyzed by qRTPCR. The results were in line with RNA-Seq(Figure 8B). Taken together, we found TGFB2 with high methylation level and low expression in ALV-J-resistant chickens from F4 generation was widespread.

\section{Effect of TGFB2 on ALV-J replication in DF-1 cells}

In order to understand the reason of TGFB2 with different methylation levels and expression levels, we evaluated the function of TGFB2 in ALV-J replication. We constructed the eukaryotic expression plasmids for pRK5-flag-TGFB2 and transfected into DF-1 cells. The viral title during the seven days after inoculation with ALV-J demonstrated that the replication of ALV-J were significant promoted in the pRK5-flag-TGFB2-transfected group compared to the control group(Fig.7A\&B). Meanwhile, to identify whether low expression of TGFB2 would repress replication of ALV-J in DF-1 cells, we transfected DF-1 cells with si-RNA for TGFB2. The viral titer for 7 days after inoculation with ALV-J showed that the replication of ALV-J were 
significant repressed in the DF-1 cells transfected with si-RNA for TGFB2, but not siNC(Fig.7C\&D). These results demonstrated the role of TGFB2 in ALV-J replication.

\section{Discussion}

Disease resistance is one of the most challenging characteristics in poultry industry. In the process of domestication and genetic selection, different chicken breeds showed different levels of resistance to pathogens. The Fayoumi chicken, which has been demonstrated to resist viral infections including Marek's disease virus [36] and avian influenza virus (AIV)[37]. ALV-J is associated with diverse pathotypes and can result in enormous economic losses, but there is no effective vaccine against ALV-J infection. Breeding chickens that are naturally resistant to infection by ALV-J is an effective control measure. In this work, eight Chinese broiler lines were used, and we had successfully identified a chicken line that has high congenital resistance to ALV-J infection. After each generation of screening, cultivation, and constant elimination, we got the chickens which resistant to ALV-J infection.

DNA methylation is a heritable modification that occurs in most eukaryotic organism, and plays a crucial role in transcriptional regulation. The role of DNA methylation in controlling the activities of gene promoters had been extensively investigated over the last three decades. So far, aberrant promoter DNA hypermethylation led to gene silence has been acceptable to most people. But the function of gene body DNA methylation is poorly understood, although several recent studies have begun to investigate the potential for how gene body DNA methylation impacts gene expression [38,39]. In the last few years, epigenetic regulation of genes had been proved was important for hypertrophic growth in chicken adipose development [40], but there is few studied analyzed the role of DNA methylation in disease resistance of chickens.

The stability of DNA methylation plays an important role in preventing tumorigenesis and disease progression. To improve our understanding of the relationship between DNA methylation and breed-specific disease resistance, we 
examined whole-genome DNA-methylation patterns in three ALV-J-resistant and susceptible-chicken samples by MeDIP-Seq, respectively. Our findings provided a comprehensive and detailed picture of DNA-methylation patterns and gene-expression levels between ALV-J-resistant and -susceptible chickens. Compared to traditional methods for identifying DMRs, MeDIP-Seq has sufficient depth and high resolution, and more DMRs were detected. Moreover, bisulfite-sequencing analysis of TGFB2 was in line with MeDIP-Seq results, which proved the high accuracy of the high-throughput sequencing technique.

The interaction of genes and environment which could influence the phenotype of chickens, including disease resistant. Under the control of resistant genes, chickens in the same environment exhibit varying degrees of resistance to ALV-J in this work. So far, there is few studies have analyzed the resistance genes in poultry. DNA methylation can affect the expression of resistance genes, thereby influencing the resistance of the chickens to ALV-J. Thus, MeDIP-Seq and RNA-Seq were performed in this study. Because the next generation of sequencing technology has made great progress, MeDIP-Seq and RNA-seq can offer higher resolution, less noise, and greater coverage results $[41,42,43]$. A comparative analysis of MeDIP-seq and RNA-seq not only might provide more information about genomewide epigenetic regulation in gene expression, but also could help screen for resistance genes against ALV-J, or methylation markers for marker-assisted selection. In turn, resistance gene or methylation markers could improve the efficiency and accuracy of stock selection, providing a reference for resistance breeding against ALV-J.

Transforming growth factor $\beta 2$ (TGFB2), one of the isoforms of TGF- $\beta$, was reported to be associated with various neoplasms $[44,45,46]$. TGFB2 can initiate an autocrine loop that promotes its own expression and enables oncogenic activity. In gliomas, the expression levels of TGFB2 are used to evaluate stages of tumor progression $[47,48]$. Recently, the important role for the TGFB2/Smad3 pathway in mediating ovarian oncogenesis was uncovered: TGFB2 enhanced metastatic ovariancancer potential by inducing Smad3-dependent epithelial-to-mesenchymal transition [49]. In prostate cancer, a quantitative increase in the promoter-methylation levels of 
TGF $\beta 2$ is associated with PCa progression [50]. In this study, ALV-J-resistant chickens with high level of TGFB2 promoter methylation than ALV-J-susceptible chickens and the DNA methylation of the TGFB2 promoter has a certain impact on TGFB2 gene expression. In addition, TGFB2 played an important role in ALV-J replication in vitro had been verified. So we suggested that the TGFB2 genes was a marker gene for selection of ALV-J-resistant chickens. A growing number of studies that indicated DNA methylation at candidate gene loci can be potential biomarkers, and this method is more common in all kinds of research [51], so we hypothesized that hypermethylated promoters of TGFB2 could use as a methylation marker to screen ALV-J-resistant chickens.

\section{Conclusion}

In conclusion, after a long period of breeding, we screened ALV-J-resistant and susceptible chickens. MeDIP-Seq and RNA-Seq in ALV-J-susceptible and -resistant chickens were preformed to explore genes which played important roles in disease resistance to ALV-J infection in chickens. TGFB2, which with high level of promoter methylation and low expression in ALV-J-resistant chickens and play an important role in ALV-J replication in vitro, was a potential marker gene for selection of ALV-Jresistant chickens.

\section{List of Abbreviations}

ALV-J: Avian leukosis virus subgroup J; MeDIP-Seq: methylated DNA immunoprecipitation sequencing; RNA-Seq: quantitative measurements of transcriptomes; DMRs: differentially methylated regions; GO analysis: gene ontology analysis; TMEM104: transmembrane protein 104; FAM173A: family with sequence similarity 173, member A; CNP1: C-type natriuretic peptide 1; HSD17B7: hydroxysteroid 17-beta dehydrogenase 7; SUPV3L1: Suv3 like RNA helicase; TGFB2: transforming growth factor beta 2; DEGs: differentially expressed genes; qRT-PCR: 
quantitative real time Polymerase Chain Reaction;

\section{Declarations}

\section{Ethics approval and consent to participate}

The animal study protocol was approved by the South China Agricultural University Committee of Animal Experiments (approval ID: SYXK-2019-0136). The protocol and the Guide for the Care and Use of Laboratory Animals established by the National Institutes of Health were closely followed in the experiments.

\section{Consent for publication}

Not applicable.

\section{Availability of data and materials}

All data generated or analyzed during this study are included in this published article and its supplementary information files. The gene expression data and DNAmethylation data can be found in GEO (accession GSE163135 and GSE143336).

\section{Competing interests}

The authors declare that they have no competing interests.

\section{Funding}

Funding for the project was provided by National Natural Science Foundation of China (grant no. 31672564, 31602053, and 31972659), and the International Science and Technology Cooperation Project of Guangdong Province (grant no. 2018B030315009). .The funding bodys had no role in the design of the study, collection, analysis, or interpretation of data, or in preparation of the manuscript.

\section{Authors' contributions}


Yiming Yan: Conceptualization, Methodology, Formal Analysis, Investigation, Writing - Original Draft, Writing - Review and Editing, Visualization. Huihua Zhang: Formal Analysis, Data Curation, Visualization. Shuang Gao: Formal Analysis, Investigation, Visualization. Huanmin Zhang: Supervision, Writing - Original Draft. Xinheng Zhang: Resources. Weiguo Chen: Investigation. Wencheng Lin: Writing Review and Editing, Resources. Qingmei Xie: Conceptualization, Supervision, Funding Acquisition, Project Administration.

\section{Acknowledgements}

Not applicable.

\section{References}

1. Payne, L.N., Venugopal, K., 2000. Neoplastic diseases: Marek's disease, avian leukosis and reticuloendotheliosis. Rev Sci Tech. 19, 544.

2. Nakamura, S., Ochiai, K., Ochi, A., Yabushita, H., Abe, A., Kishi, S., Sunden, Y., Umemura, T., 2014. Cardiac pathology and molecular epidemiology by avian leukosis viruses in Japan. Plos One. 9, e86546.

3. Payne, L.N., Nair, V., 2012. The long view: 40 years of avian leukosis research. Avian Pathol. 41, 11-19.

4. Payne, L.N., Brown, S.R., Bumstead, N., Howes, K., Frazier, J.A., Thouless, M.E., 1991. A novel subgroup of exogenous avian leukosis virus in chickens. J Gen Virol. 72, 801-807.

5. Payne, L.N., Gillespie, A.M., Howes, K., 1993. Unsuitability of chicken sera for detection of exogenous ALV by the group-specific antigen ELISA. Vet Rec. 132, $555-557$.

6. Du, Y., Cui, Z., Qin, A., 1999. Subgroup J avian leukosis viruses in China. China Poult. Sci. 3, 1-4. 
7. Fadly, A.M., Smith, E.J., 1999. Isolation and some characteristics of a subgroup Jlike avian leukosis virus associated with myeloid leukosis in meat-type chickens in the United States. Avian Dis. 43, 391-400.

8. Chen, W., Liu, Y., Li, H., Chang, S., Shu, D., Zhang, H., Chen, F., Xie, Q. 2015. Intronic deletions of tva receptor gene decrease the susceptibility to infection by avian sarcoma and leukosis virus subgroup A. Scientific Reports. 5, 9900.

9. Chen, W., Liu, Y., Li, A., Li, X., Li, H., Dai, Z., Yan, Y., Zhang, X., Shu, D., Zhang, H., Lin, W., Ma, J., Xie, Q., 2017. A premature stop codon within the tvb receptor gene results in decreased susceptibility to infection by avian leukosis virus subgroups B, D, and E. Oncotarget. 8, 105942-105956.

10. Cheng, H.H., Kaiser, P., Lamont, S.J., 2013. Integrated Genomic Approaches to Enhance Genetic Resistance in Chickens. Annu Rev Anim Biosci. 1, 239-260.

11. Li, X., Chen, W., Zhang, H., Li, A., Shu, D., Li, H., Dai, Z., Yan, Y., Zhang, X., Lin, W., Ma, J., Xie, Q., 2018. Naturally Occurring Frameshift Mutations in the tvb Receptor Gene Are Responsible for Decreased Susceptibility of Chicken to Infection with Avian Leukosis Virus Subgroups B, D, and E. J Virol. 92, e0177017.

12. Abhishek, S., and Palamadai, K.S., 2016. Epidermal Differentiation Complex: A Review on Its Epigenetic Regulation and Potential Drug Targets. Cell J. 18, 1-6.

13. Douvaras, P., Rusielewicz, T., Kim, K., Haines, J., Casaccia, P., Fossati, V., 2016. Epigenetic Modulation of Human Induced Pluripotent Stem Cell Differentiation to Oligodendrocytes. Int J Mol Sci. 17, 614.

14. Liu, Y., Giannopoulou, E.G., Wen, D., Falciatori, I., Elemento, O., Allis, C.D., Rafii, S., Seandel, M., 2016. Epigenetic profiles signify cell fate plasticity in unipotent spermatogonial stem and progenitor cells. Nat Commun. 7, 11275. 
15. Dawson, M.A., Kouzarides, T., 2012. Cancer Epigenetics: From Mechanism to Therapy. Cell. 150, 12-27.

16. Goeppert, B., Konermann, C., Schmidt, C.R., Bogatyrova, O., Geiselhart, L., Ernst, C., Gu, L., Becker, N., Zucknick, M., Mehrabi, A., Hafezi, M., Klauschen, F., Stenzinger, A., Warth, A., Breuhahn, K., Renner, M., Weichert, W., Schirmacher, P., Plass, C., Weichenhan, D., 2014. Global alterations of DNA methylation in cholangiocarcinoma target the Wnt signaling pathway. Hepatology. 59, 544-54.

17. Jaenisch, R., Bird, A., 2003. Epigenetic regulation of gene expression: how the genome integrates intrinsic and environmental signals. Nat Genet. 33 Suppl, 245254.

18. Kuratomi, G., Iwamoto, K., Bundo, M., Kusumi, I., Kato, N., Iwata, N., Ozaki, N., Kato, T., 2008. Aberrant DNA methylation associated with bipolar disorder identified from discordant monozygotic twins. Mol Psychiatr. 13, 429-441.

19. Petronis, A., 2010. Epigenetics as a unifying principle in the aetiology of complex traits and diseases. Nature. 465, 721-727.

20. Weber, M., Hellmann, I., Stadler, M.B., Ramos, L., Pääbo, S., Rebhan, M., Schübeler, D., 2007. Distribution, silencing potential and evolutionary impact of promoter DNA methylation in the human genome. Nat Genet. 39, 457-466.

21. Zochbauer-Muller, S., Fong, K.M., Maitra, A., Lam, S., Geradts, J., Ashfaq, R., Virmani, A.K., Milchgrub, S., Gazdar, A.F., Minna, J.D., 2001. 5' CpG island methylation of the FHIT gene is correlated with loss of gene expression in lung and breast cancer. Cancer Res. 61, 3581-3585. 
22. Yang, H.J., Liu, V.W., Wang, Y., Tsang, P.C., Ngan, H.Y., 2006. Differential DNA methylation profiles in gynecological cancers and correlation with clinicopathological data. Bmc Cancer. 6, 212.

23. Wang, Y., Li, J., Cui, Y., Li, T., Ng, K.M., Geng, H., Li, H., Shu, X.S., Li, H., Liu, W., Luo, B., Zhang, Q., Mok, T.S.K., Zheng, W., Qiu, X., Srivastava, G., Yu, J., Sung, J.J.Y., Chan, A.T.C., Ma, D., Tao, Q., Han, W., 2009. CMTM3, Located at the Critical Tumor Suppressor Locus 16q22.1, Is Silenced by CpG Methylation in Carcinomas and Inhibits Tumor Cell Growth through Inducing Apoptosis. Cancer Res. 69, 5194-5201.

24. Yan, Y., Yang, N., Cheng, H.H., Song, J., Qu, L., 2015. Genome-wide identification of copy number variations between two chicken lines that differ in genetic resistance to Marek’s disease. Bmc Genomics. 16.

25. Li, X.Y., Qu, L.J., Yao, J.F., Yang, N., 2006. Skewed allele frequencies of an Mx gene mutation with potential resistance to avian influenza virus in different chicken populations. Poult Sci. 85, 1327-9.

26. Sandford, E.E., Orr, M., Shelby, M., Li, X., Zhou, H., Johnson, T.J., Kariyawasam, S., Liu, P., Nolan, L.K., Lamont, S.J., 2012. Leukocyte transcriptome from chickens infected with avian pathogenic Escherichia coli identifies pathways associated with resistance. Results in Immunology. 2, 44-53.

27. Li, X., Swaggerty, C.L., Kogut, M.H., Chiang, H.I., Wang, Y., Genovese, K.J., He, H., Zhou, H., 2010. Gene expression profiling of the local cecal response of genetic chicken lines that differ in their susceptibility to Campylobacter jejuni colonization. Plos One. 5, e11827.

28. Zhang, X., Yan, Y., Lin, W., Li, A., Zhang, H., Lei, X., Dai, Z., Li, X., Li, H., Chen, W., Chen, F., Ma, J., Xie, Q., 2019. Circular RNA Vav3 sponges gga-miR375 to promote epithelial-mesenchymal transition. RNA Biol. 16, 118-132. 
29. Langmead, B., Salzberg, S.L., 2012. Fast gapped-read alignment with Bowtie 2. Nat Methods. 9, 357-359.

30. Zhang, Y., Liu, T., Meyer, C.A., Eeckhoute, J., Johnson, D.S., Bernstein, B.E., Nusbaum, C., Myers, R.M., Brown, M., Li, W., Liu, X.S., 2008. Model-based analysis of ChIP-Seq (MACS). Genome Biol. 9, R137.

31. Shen, L., Shao, N.Y., Liu, X., Maze, I., Feng, J., Nestler, E.J., 2013. diffReps: detecting differential chromatin modification sites from ChIP-seq data with biological replicates. Plos One. 8, e65598.

32. Trapnell, C., Williams, B.A., Pertea, G., Mortazavi, A., Kwan, G., van Baren, M.J., Salzberg, S.L., Wold, B.J., Pachter, L., 2010. Transcript assembly and quantification by RNA-Seq reveals unannotated transcripts and isoform switching during cell differentiation. Nat Biotechnol. 28, 511-515.

33. Zhang, H., Hao, Y., Yang, J., Zhou, Y., Li, J., Yin, S., Sun, C., Ma, M., Huang, Y., Xi, J.J., 2011. Genome-wide functional screening of miR-23b as a pleiotropic modulator suppressing cancer metastasis. Nat Commun. 2, 554.

34. Yu, G., Wang, L., Han, Y., He, Q., 2012. clusterProfiler: an R Package for Comparing Biological Themes Among Gene Clusters. OMICS: A Journal of Integrative Biology. 16, 284-287.

35. Yu, G., Wang, L., Yan, G., He, Q., 2015 . DOSE: an R/Bioconductor package for disease ontology semantic and enrichment analysis. Bioinformatics. 31, 608-609.

36. Lakshmanan, N.; Gavora, J.S.; Lamont, S.J., 1997. Major histocompatibility complex class II DNA polymorphisms in chicken strains selected for Marek's disease resistance and egg production or for egg production alone. Poult Sci. 76, 1517-23.

37. Wang, Y., Lupiani, B., Reddy, S.M., Lamont, S.J., Zhou, H., 2014. RNA-seq analysis revealed novel genes and signaling pathway associated with disease resistance to avian influenza virus infection in chickens. Poult Sci. 93, 485-93. 
38. Kulis, M., Heath, S., Bibikova, M., Queirós, A.C., Navarro, A., Clot, G., Martínez-Trillos, A., Castellano, G., Brun-Heath, I., Pinyol, M., Barberán-Soler, S., Papasaikas, P., Jares, P., Beà, S., Rico, D., Ecker, S., Rubio, M., Royo, R., Ho, V., Klotzle, B., Hernández, L., Conde, L., López-Guerra, M., Colomer, D., Villamor, N., Aymerich, M., Rozman, M., Bayes, M., Gut, M., Gelpí, J.L., Orozco, M., Fan, J., Quesada, V., Puente, X.S., Pisano, D.G., Valencia, A., LópezGuillermo, A., Gut, I., López-Otín, C., Campo, E., Martín-Subero, J.I., 2012.Epigenomic analysis detects widespread gene-body DNA hypomethylation in chronic lymphocytic leukemia. Nat Genet. $\quad 44,1236-1242$.

39. Yang, X., Han, H., De Carvalho, D.D., Lay, F.D., Jones, P.A., Liang, G., 2014. Gene Body Methylation Can Alter Gene Expression and Is a Therapeutic Target in Cancer. Cancer Cell. 26, 577-590.

40. Sun, Y., Li, R., Zhai, G., Zhang, X., Wang, Y., 2019. DNA methylation of the promoter downregulates expression in chicken lines. Arch Anim Breed. 62, 375382.

41. Down, T.A., Rakyan, V.K., Turner, D.J., Flicek, P., Li, H., Kulesha, E., Graf, S., Johnson, N., Herrero, J., Tomazou, E.M., Thorne, N.P., Backdahl, L., Herberth, M., Howe, K.L., Jackson, D.K., Miretti, M.M., Marioni, J.C., Birney, E., Hubbard, T.J., Durbin, R., Tavare, S., Beck, S., 2008. A Bayesian deconvolution strategy for immunoprecipitation-based DNA methylome analysis. Nat Biotechnol. 26, 779-785.

42. Carrier, G., Santoni, S., Rodier-Goud, M., Canaguier, A., Kochko, A.D., Dubreuil-Tranchant,C., This, P., Boursiquot, J.M., Cunff, L.L. 2011. An efficient and rapid protocol for plant nuclear DNA preparation suitable for next generation sequencing methods. Am J Bot. 98, e13-e15. 
43. De Luca, A., Roma, C., Gallo, M., Fenizia, F., Bergantino, F., Frezzetti, D., Costantini, S., Normanno, N., 2014. RNA-seq analysis reveals significant effects of EGFR signalling on the secretome of mesenchymal stem cells. Oncotarget. 5, 10518-10528.

44. Joseph, J.V., Conroy, S., Tomar, T., Eggens-Meijer, E., Bhat, K., Copray, S., Walenkamp, A.M.E., Boddeke, E., Balasubramanyian, V., Wagemakers, M., den Dunnen, W.F.A., Kruyt, F.A.E., 2014. TGF- $\beta$ is an inducer of ZEB1dependent mesenchymal transdifferentiation in glioblastoma that is associated with tumor invasion. Cell Death Dis. 5, e1443.

45. Niu, G.,Li, B.,Sun, L.,An, C., 2015. MicroRNA-153 Inhibits Osteosarcoma Cells Proliferation and Invasion by Targeting TGF- $\beta 2$. Plos One. 10, e0119225.

46. Lu, R., Ji, Z., Li, X., Qin, J., Cui, G., Chen, J., Zhai, Q., Zhao, C., Zhang, W., Yu, Z., 2015. Tumor suppressive microRNA-200a inhibits renal cell carcinoma development by directly targeting TGFB2. Tumor Biology. 36, 6691-6700.

47. Jachimczak, P., Hessdorfer, B., Fabel-Schulte, K., Wismeth, C., Brysch, W., Schlingensiepen, K.H., Bauer, A., Blesch, A., Bogdahn, U., 1996. Transforming growth factor-beta-mediated autocrine growth regulation of gliomas as detected with phosphorothioate antisense oligonucleotides. Int J Cancer. 65, 332-337.

48. Kjellman, C., Olofsson, S.P., Hansson, O., Von Schantz, T., Lindvall, M., Nilsson, I., Salford, L.G., Sjogren, H.O., Widegren, B., 2000. Expression of TGFbeta isoforms, TGF-beta receptors, and SMAD molecules at different stages of human glioma. Int J Cancer. 89, 251-258.

49. Do, T.V., Kubba, L.A., Du, H., Sturgis, C.D., Woodruff, T.K., 2008. Transforming Growth Factor- 1, Transforming Growth Factor- 2, and Transforming Growth Factor- 3 Enhance Ovarian Cancer Metastatic Potential by 
Inducing a Smad3-Dependent Epithelial-to-Mesenchymal Transition. Mol Cancer Res. 6, 695-705.

50. Liu, L., Kron, K.J., Pethe, V.V., Demetrashvili, N., Nesbitt, M.E., Trachtenberg, J., Ozcelik, H., Fleshner, N.E., Briollais, L., van der Kwast, T.H., Bapat, B., 2011. Association of tissue promoter methylation levels of APC, TGFbeta2, HOXD3 and RASSF1A with prostate cancer progression. Int J Cancer. 129, 24542462.

51. Varela-Rey, M., Woodhoo, A., Martinez-Chantar, M.L., Mato, J.M., Lu, S.C., 2013. Alcohol, DNA methylation, and cancer. Alcohol Res. 35, 25-35.

\section{Supplementary data}

S1 Fig: Distribution of MeDIP-Seq reads on each chromosome in each sample; S1 Table: Results of infection status of F4 generation; S2 Table: Total observerd DMRs in ALV-J-susceptible chickens and ALV-J-resistant chickens; S3 Table: Significantly enriched GO categories for DMRs; S4 Table: Significantly enriched pathways for DMRs; S5 Table: Total observerd DEGs in ALV-J-susceptible chickens and ALV-J-resistant chickens; S6 Table: Significantly enriched GO categories for DEGs; S7 Table: Significantly enriched pathways for DEGs; S8 Table: Integrated analysis of MeDIP-seq and RNA-seq data; S9 Table: Information regarding the primers used to validate the methylation state of target gene promoter region by BSP. S10 Table: Table S10.Primers for qRT-PCR validation.

\section{Figure legend}


Figure 1. Character of ALV-J-resistant and ALV-J-susceptible chickens' samples. (A) Spleens of ALV-J-susceptible(left) and ALV-J-resistant chickens(right). (B) Immunofluorescence showed positive green fluorescence signals in DF-1 cells inoculated liver homogenate of ALV-J-susceptible chickens(a) and no fluorescent signals in DF-1 cells inoculated liver homogenate of ALV-J-resistant chickens(b).

Figure 2. Distribution of methylation peaks in different genic regions of each sample, including promoter, intro, exon, intergenic and upstream regions. (A-C) were ALV-J-resistant chickens and (D-E) were ALV-J-susceptible chickens. Similar to the genic distribution of methylation peaks, the DMRs were most often located in the intergenic region in both groups.

Figure 3. Distribution of hypermethylation and hypomethylation in each gene elements of ALV-J-susceptible chicken samples vs. ALV-J-resistant chicken samples.

Figure 4. Validation of hypomethylated gene TGFB2 in ALV-J-susceptible chicken samples vs. ALV-J-resistant chicken samples by bisulfite sequencing. The validation result of the DMR of TGFB2 in promoter region by bisulfate sequencing is showed. The filled circles represent the methylated $\mathrm{CpG}$ locus and the hollow circles represent the unmethylated $\mathrm{CpG}$ locus. The Chi-square test showed the differences between ALV-J-resistant and ALV-J-susceptible chickens.

Figure 5. Gene ontology and pathway analysis of the hypermethylated DMGs and hypomethylated DMGs. DAVID software was used to conduct the analysis and partial results were shown in this figure. (A) The significant GO categories of 
hypermethylated DMRs in ALV-J-resistant chickens compared with ALV-Jsusceptible chickens $(\mathrm{P}<0.05)$. (B) The significant GO categories of hypomethylated DMRs in ALV-J-resistant chickens compared with ALV-J-susceptible chickens $(\mathrm{P}<0.05)$.

Figure 6. General profiling of the differentially expressed genes (DEGs). (A) Heatmap of DEGs from RNA-Seq profiles. (B) The significant GO categories of of DEGs. (C) Pathways analysis of the DEGs. (D) Validation of differentially expressed genes by qRT-PCR. The vertical axis denotes fold change of the RNA-seq and qRTPCR in the ALV-J-susceptible chickens compared to the ALV-J-resistant chickens.

Figure 7. Integrated analysis of MeDIP-seq and RNA-seq data. (A) Venn diagram of DEGs and DMGs. Each group was divided into up-regulated and downregulated subgroups. The number of genes is given in the middle of each figure section. (B) Bidirectional expression patterns of differentially hypermethylated genes with various genomic elements. (C) Differentially hypomethylated genes with various genomic elements. The number of genes is given at the top of each graph bar.

Figure 8. Identification of TGFB2 methylation level and expression in randomly selected chickens by BSP and qRT-PCR. (A) The methylation status of TGFB2 in promoter region was investigated among randomly selected chickens by BSP. (B) Relative expression of TGFB2 in randomly selected chickens. Data shown are the means $\pm \mathrm{SE}, * \mathrm{P}<0.05$ and $* * \mathrm{P}<0.01$ 
Figure 9. Effects of TGFB2 on ALV-J replication. (A) Western blotting of the proteins isolated $24 \mathrm{~h}$ after transfection of DF-1 cells with pRK5-flag-TGFB2 or pRK5-flag, and for the normal cells group. (B) Proliferation curve of ALV-J in DF-1 cells transfected with pRK5-flag-TGFB2 or pRK5-flag and challenged with ALV-J. (C) Western blotting of the proteins isolated 24h after transfection of DF-1 cells with siRNA targeted to TGFB2 or si-NC, and for the normal cells group. (D) Proliferation curve of ALV-J in DF-1 cells transfected with the siRNA for TGFB2 or si-NC and challenged with ALV-J; $* \mathrm{P}<0.05, * * \mathrm{P}<0.01$ 
Table 1. Number of reads generated by MeDIP-seq for each sample

\begin{tabular}{ccccc}
\hline Sample & Raw reads & Clean reads & Mapped to reference Genome & Mapped percentage \\
\hline 3 & $17,108,942$ & $17,101,214$ & $14,360,967$ & $83.98 \%$ \\
4 & $17,719,218$ & $17,708,194$ & $14,779,696$ & $83.46 \%$ \\
26 & $17,343,579$ & $17,329,183$ & & $82.33 \%$ \\
8 & $16,993,746$ & $16,982,842$ & $14,267,708$ & $82.72 \%$ \\
9 & $17,977,748$ & $17,965,218$ & $14,048,795$ & $83.10 \%$ \\
& & & & $82.13 \%$ \\
\hline
\end{tabular}

Sample numbers 3, 4 and 26 represent ALV-J-resistant chickens; 8, 9 and 14 represent ALV-J-susceptible chickens. 
Table 2. Number of reads generated by RNA-seq for each sample

\begin{tabular}{ccccc}
\hline Sample & Raw reads & Clean reads & Overall aligned reads & Overall alignment rate \\
\hline 3 & $31,972,032$ & $31,752,754$ & $27,232,317$ & $85.76 \%$ \\
4 & $29,101,802$ & $28,323,384$ & $25,108,913$ & $88.65 \%$ \\
26 & $23,346,692$ & $22,442,240$ & $17,732,522$ & $79.01 \%$ \\
8 & $28,812,098$ & $27,209,832$ & $21,958,483$ & $80.70 \%$ \\
9 & $22,150,968$ & $19,975,018$ & $16,079,228$ & $80.50 \%$ \\
14 & $33,766,962$ & $32,350,232$ & $24,801,434$ & $76.67 \%$ \\
\hline
\end{tabular}

Sample numbers 3, 4 and 26 represent ALV-J-resistant chickens; 8, 9 and 14 represent ALV-J-susceptible chickens. 
Table 3. Differentially methylated and regulated genes with methylation profiles in promoter regions.

\begin{tabular}{|c|c|c|c|c|c|c|c|}
\hline GeneID & Chromosome & $\begin{array}{l}\text { Genomic } \\
\text { context }\end{array}$ & $\begin{array}{c}\text { Methylated } \\
\text { region }\end{array}$ & $\begin{array}{l}\text { Fold change } \\
\text { (MeDIP-seq) }\end{array}$ & p-value & $\begin{array}{l}\text { Fold } \\
\text { change } \\
\text { (RNA- } \\
\text { seq) }\end{array}$ & p-value \\
\hline CNP1 & $\operatorname{chr} 1$ & promoter & $\begin{array}{l}76428141- \\
76428600\end{array}$ & 2.462 & $2.28 \mathrm{E}-01$ & -5.171 & 2.35E-02 \\
\hline FAM173A & chr14 & promoter & $\begin{array}{l}13177801- \\
13178160\end{array}$ & 2.869 & $1.77 \mathrm{E}-01$ & -3.338 & $9.10 \mathrm{E}-03$ \\
\hline TMEM104 & $\operatorname{chr} 18$ & promoter & $\begin{array}{l}10726761- \\
10727020\end{array}$ & 4.796 & $2.14 \mathrm{E}-02$ & -4.743 & $5.00 \mathrm{E}-05$ \\
\hline HSD17B7 & chr8 & promoter & $\begin{array}{l}3791861- \\
3792360\end{array}$ & -3.125 & $6.14 \mathrm{E}-06$ & 2.799 & $3.16 \mathrm{E}-02$ \\
\hline SUPV3L1 & chr6 & promoter & $\begin{array}{l}10421761- \\
10422180\end{array}$ & -2.601 & $1.89 \mathrm{E}-04$ & 2.309 & $3.06 \mathrm{E}-02$ \\
\hline TGFB2 & $\operatorname{chr} 3$ & promoter & $\begin{array}{l}18754421- \\
18754940\end{array}$ & -5.082 & $1.67 \mathrm{E}-11$ & 4.540 & $5.00 \mathrm{E}-05$ \\
\hline
\end{tabular}

The methylation and expression statuses of the ALV-J-susceptible chicken' samples were utilized as references for those in the ALV-J-resistant chicken' samples. 


\section{Figures}

A ALV-J-susceptible chicken ALV-J-resistant chicken B

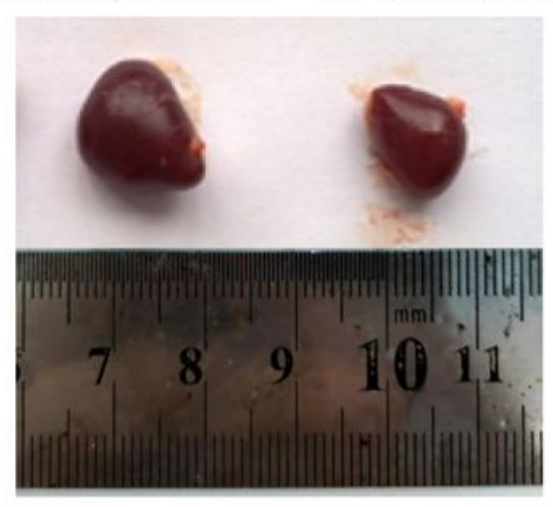

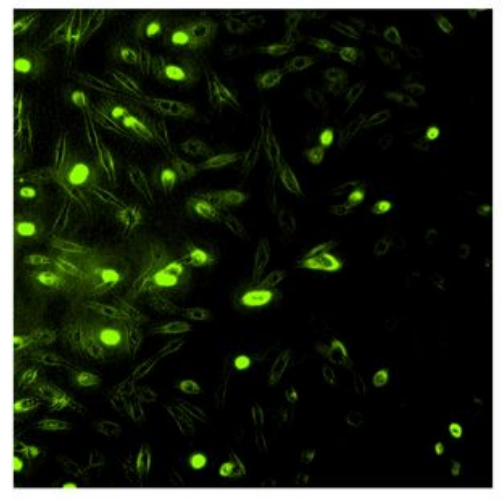

C

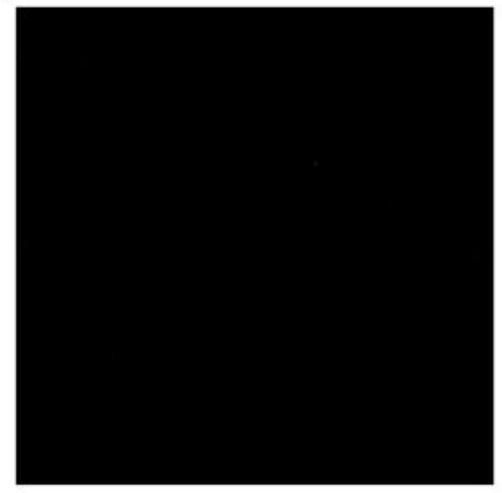

\section{Figure 1}

Character of ALV-J-resistant and ALV-J-susceptible chickens' samples. (A) Spleens of ALV-Jsusceptible(left) and ALV-J-resistant chickens(right). (B) Immunofluorescence showed positive green fluorescence signals in DF-1 cells inoculated liver homogenate of ALV-J-susceptible chickens(a) and no fluorescent signals in DF-1 cells inoculated liver homogenate of ALV-J-resistant chickens(b). 


\section{Methylated region classification}

A

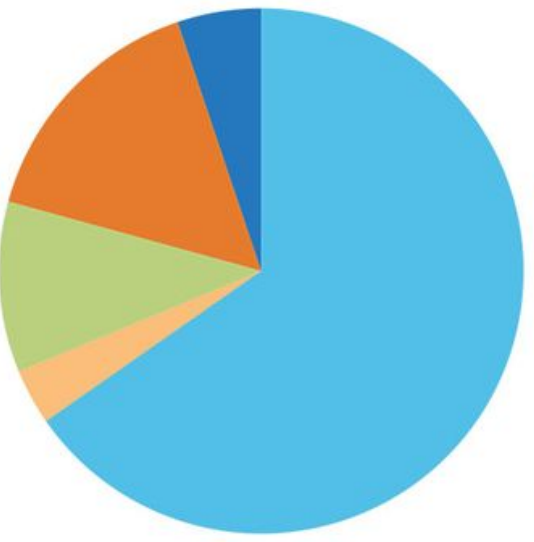

B

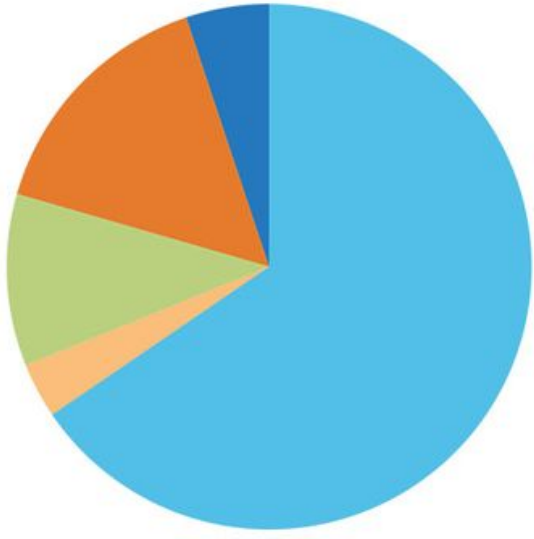

C

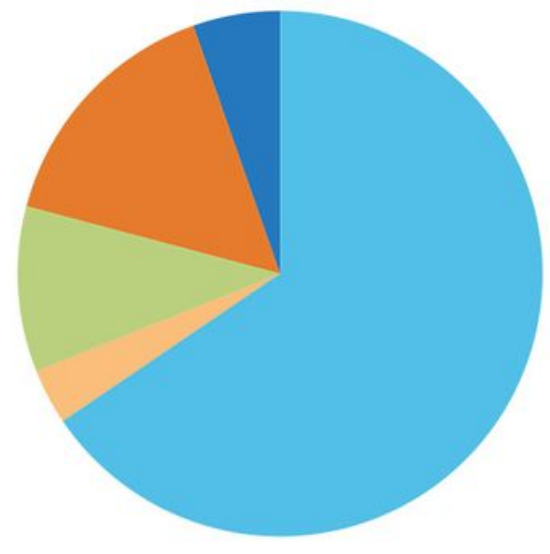

promoter $5.17 \%$

upstream $15.57 \%$

intron $10.52 \%$

exon $345 \%$

intergenic 65.30

promoter $5.12 \%$

upstream $15.41 \%$

intron $10.59 \%$

exon $3.41 \%$

intergenic $65.47 \%$

promoter $5.37 \%$

upstream $15.50 \%$

intron $10.18 \%$

exon $3.45 \%$

intergenic $65.51 \%$
D

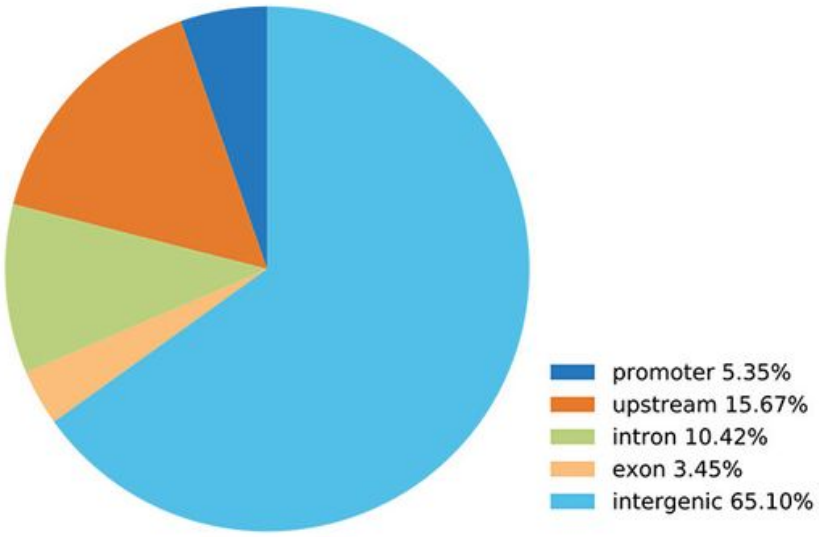

E

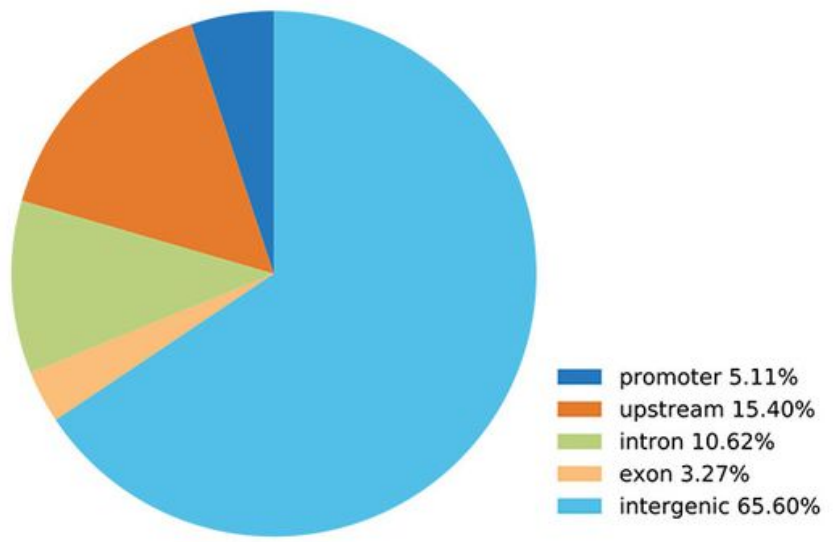

F

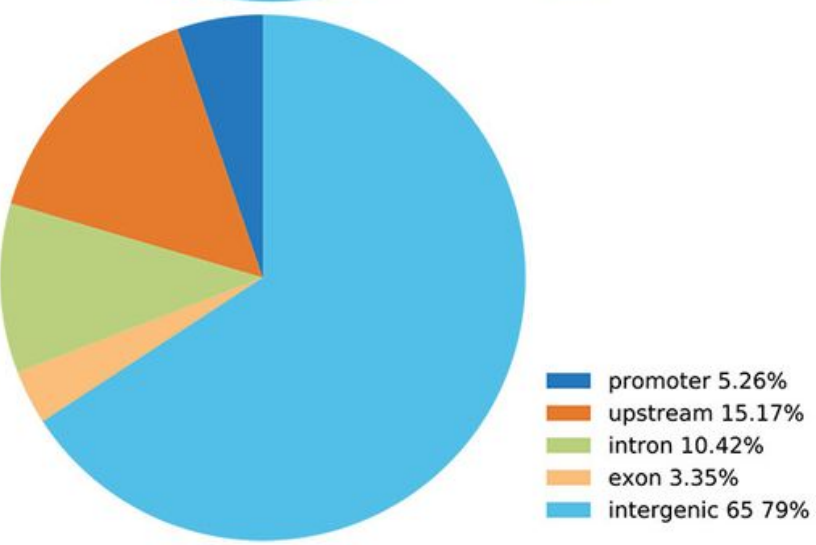

\section{Figure 2}

Distribution of methylation peaks in different genic regions of each sample, including promoter, intro, exon, intergenic and upstream regions. (A-C) were ALV-J-resistant chickens and (D-E) were ALV-Jsusceptible chickens. Similar to the genic distribution of methylation peaks, the DMRs were most often located in the intergenic region in both groups. 


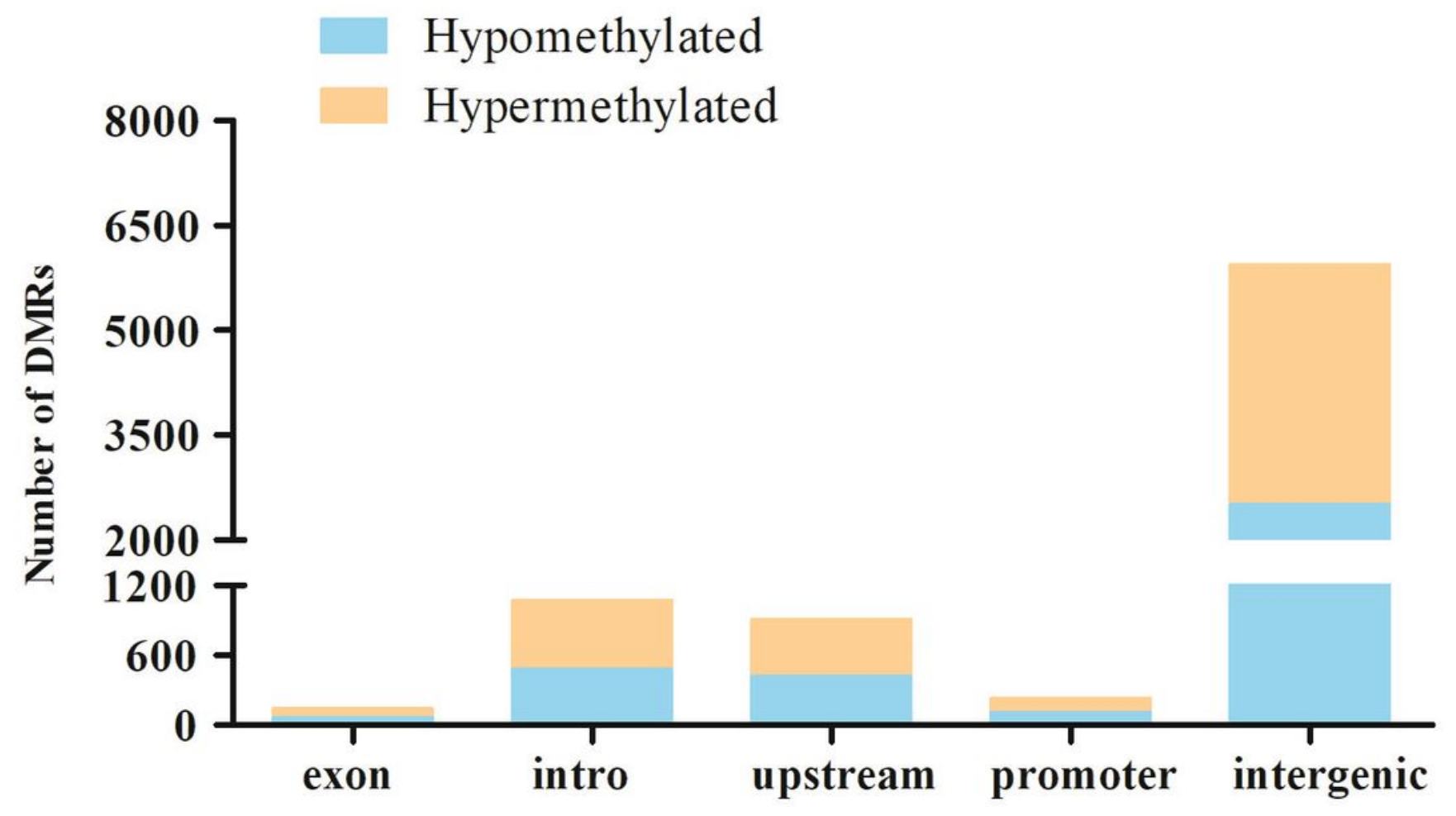

Figure 3

Distribution of hypermethylation and hypomethylation in each gene elements of ALV-J-susceptible chicken samples vs. ALV-J-resistant chicken samples.

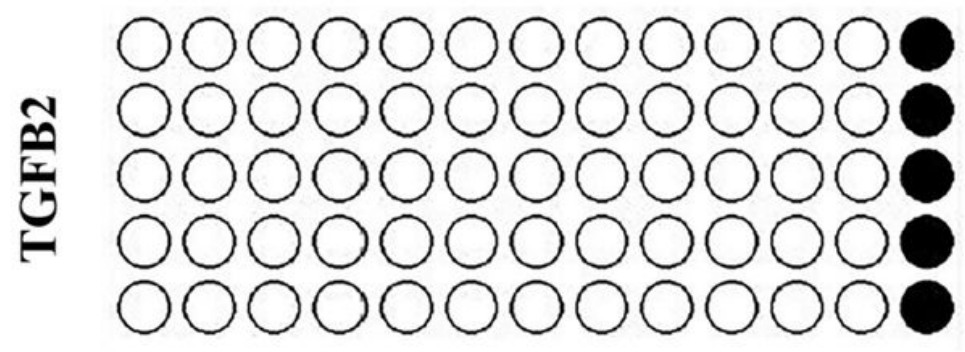

ALV-J-resistant chickens

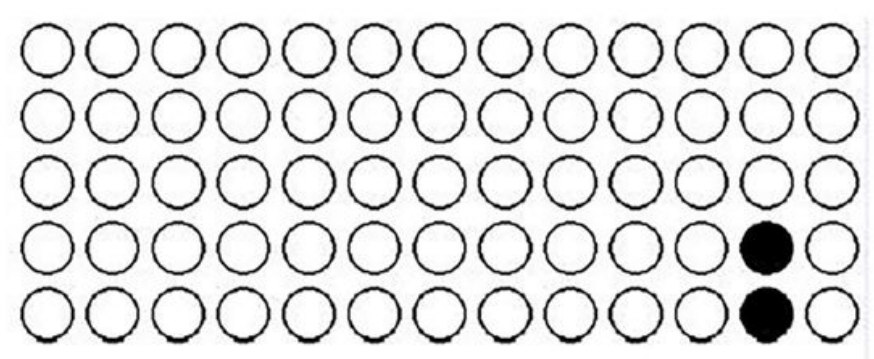

ALV-J-susceptible chickens

\section{Figure 4}

Validation of hypomethylated gene TGFB2 in ALV-J-susceptible chicken samples vs. ALV-J-resistant chicken samples by bisulfite sequencing. The validation result of the DMR of TGFB2 in promoter region by bisulfate sequencing is showed. The filled circles represent the methylated $\mathrm{CpG}$ locus and the hollow circles represent the unmethylated CpG locus. The Chi-square test showed the differences between ALV-Jresistant and ALV-J-susceptible chickens. 
A

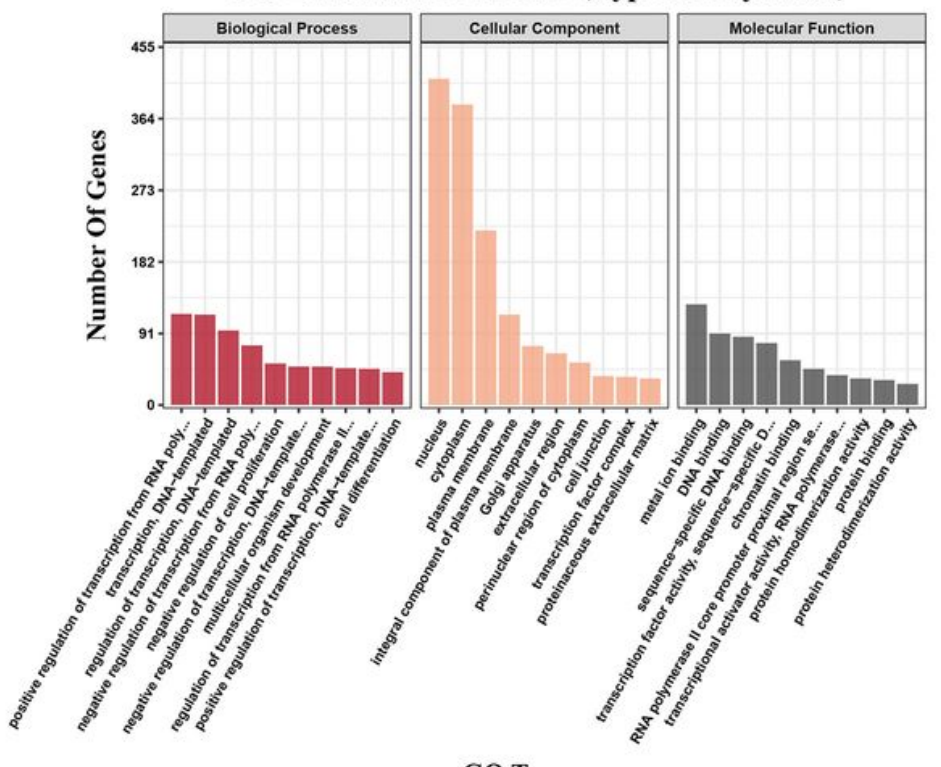

GO Term
B

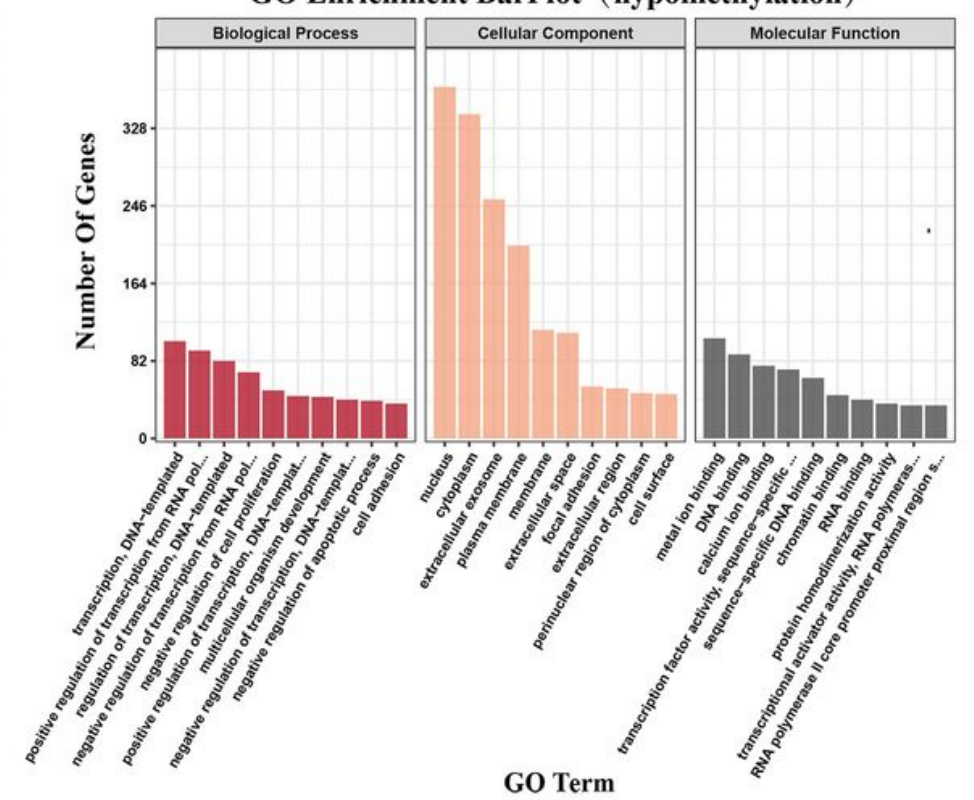

Figure 5

Gene ontology and pathway analysis of the hypermethylated DMGs and hypomethylated DMGs. DAVID software was used to conduct the analysis and partial results were shown in this figure. (A) The significant GO categories of hypermethylated DMRs in ALV-J-resistant chickens compared with ALV-Jsusceptible chickens $(P<0.05)$. (B) The significant GO categories of hypomethylated DMRs in ALV-Jresistant chickens compared with ALV-J-susceptible chickens $(P<0.05)$. 
A

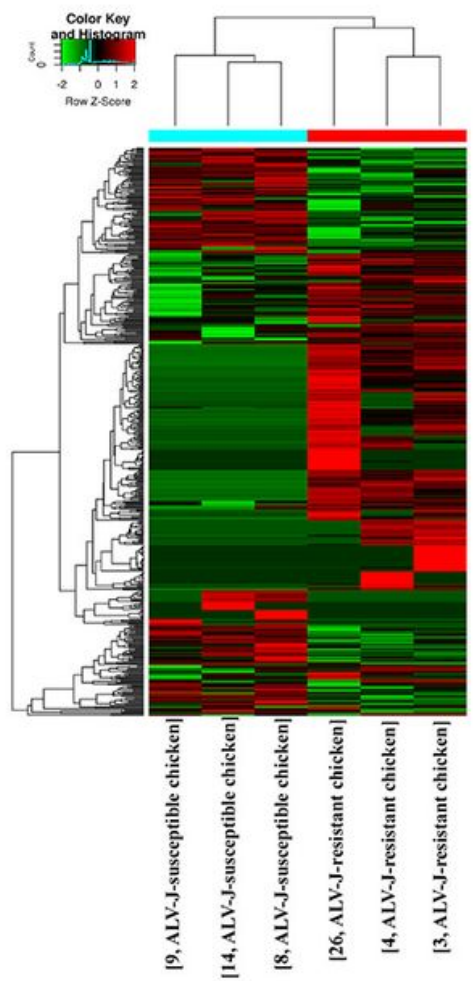

C

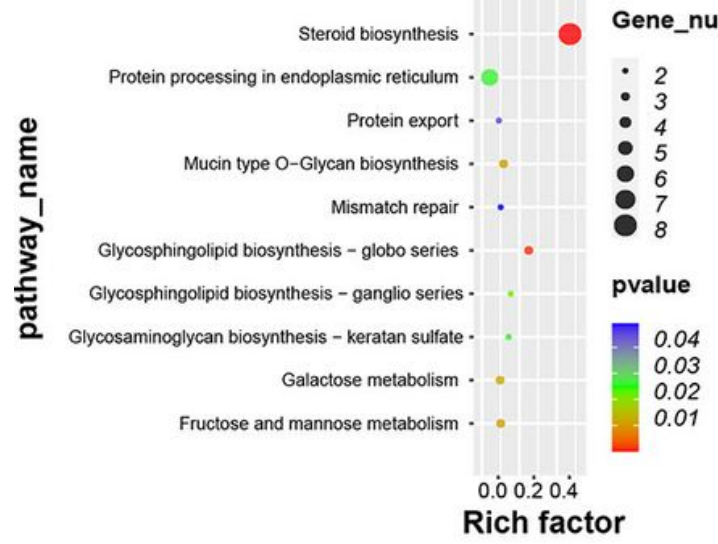

B

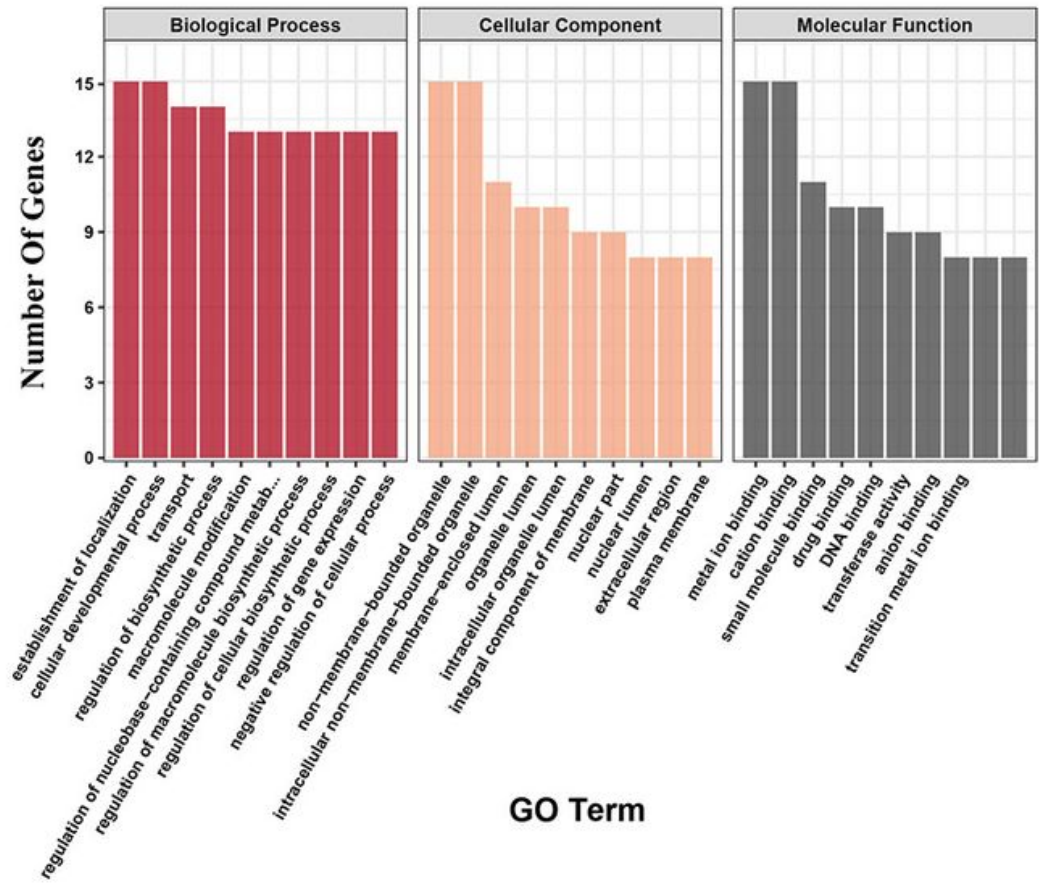

D

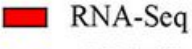

qRT-PCR

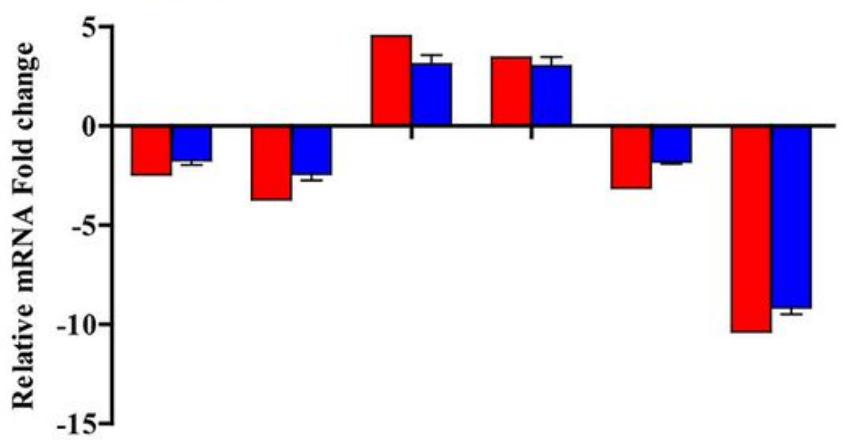

CPN1 STAT4 TGFB2 GRAP GOSR1 SSBP1

Figure 6

General profiling of the differentially expressed genes (DEGs). (A) Heatmap of DEGs from RNA-Seq profiles. (B) The significant GO categories of of DEGs. (C) Pathways analysis of the DEGs. (D) Validation of differentially expressed genes by qRT-PCR. The vertical axis denotes fold change of the RNA-seq and qRT-PCR in the ALV-J-susceptible chickens compared to the ALV-J-resistant chickens. 

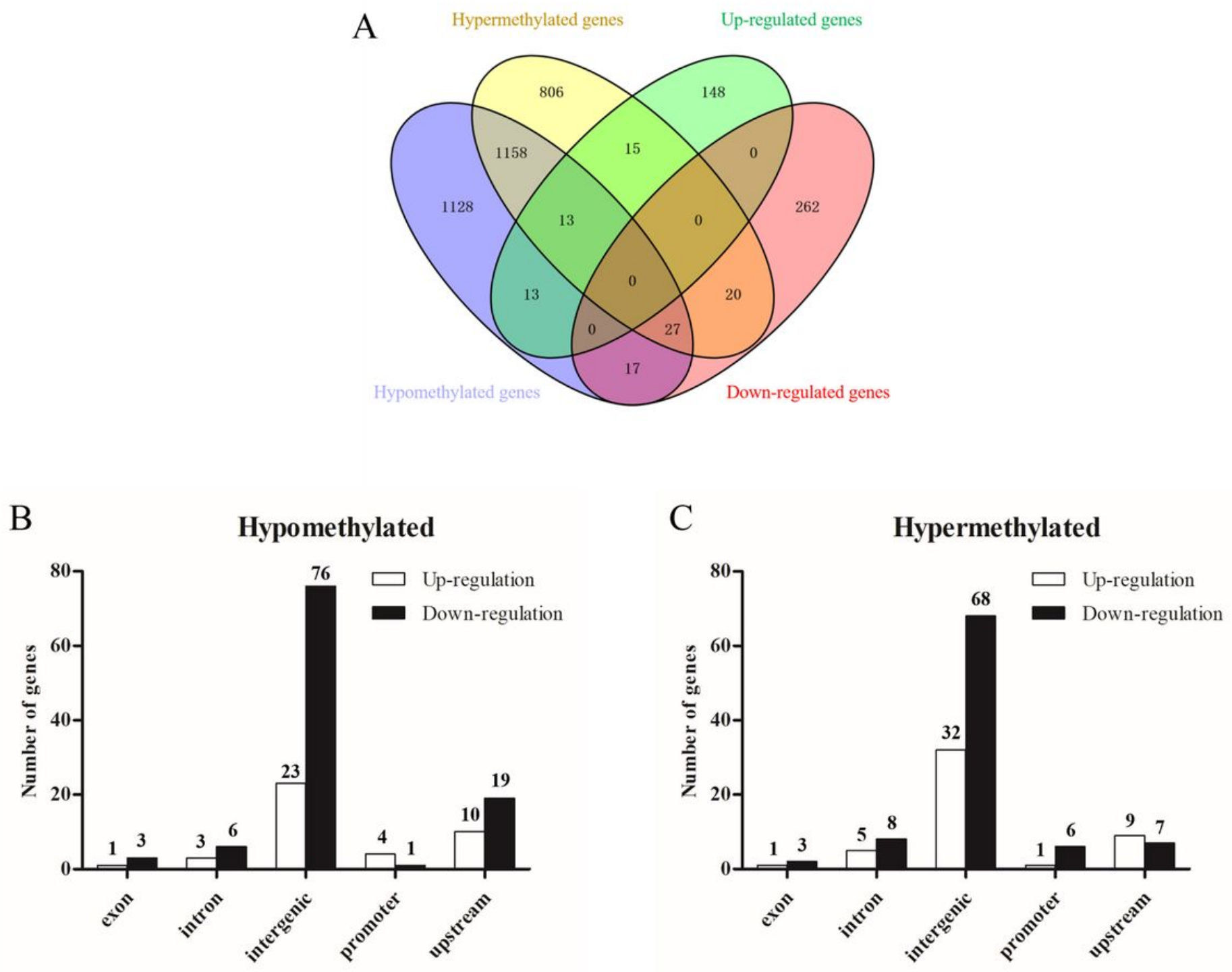

Figure 7

Integrated analysis of MeDIP-seq and RNA-seq data. (A) Venn diagram of DEGs and DMGs. Each group was divided into up-regulated and down-regulated subgroups. The number of genes is given in the middle of each figure section. (B) Bidirectional expression patterns of differentially hypermethylated genes with various genomic elements. (C) Differentially hypomethylated genes with various genomic elements. The number of genes is given at the top of each graph bar. 


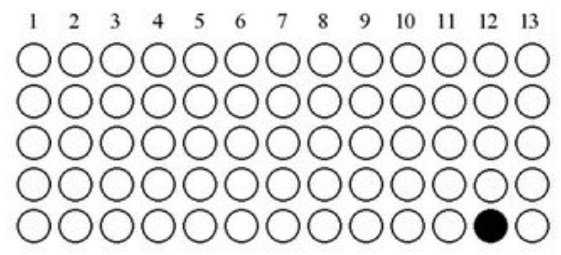

궁

$\begin{array}{lllllllllllll}1 & 2 & 3 & 4 & 5 & 6 & 7 & 8 & 9 & 10 & 11 & 12 & 13\end{array}$

0000000000000

0000000000000 0000000000000

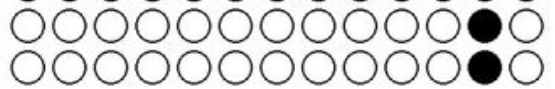

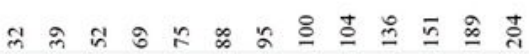

$\begin{array}{lllllllllllll}1 & 2 & 3 & 4 & 5 & 6 & 7 & 8 & 9 & 10 & 11 & 12 & 13\end{array}$

0000000000000

0000000000000 0000000000000 0000000000000 0000000000000

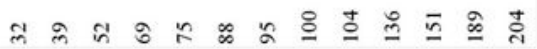

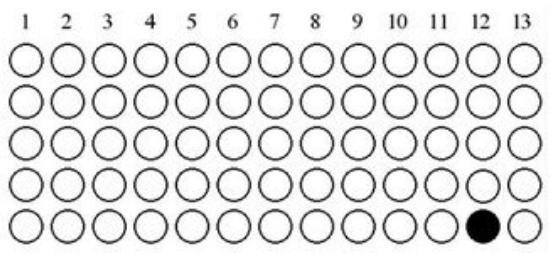

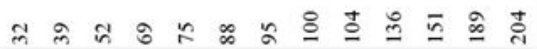

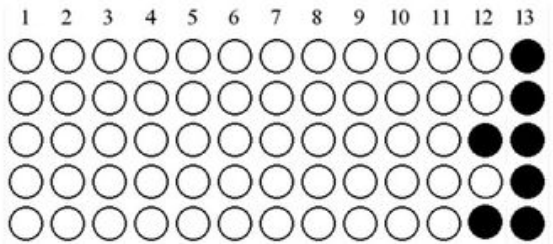

ल

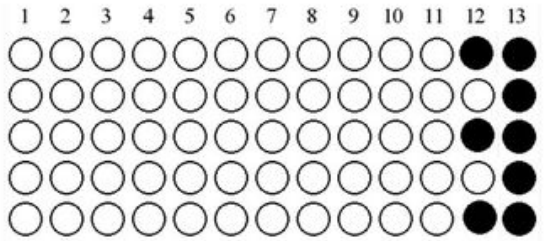

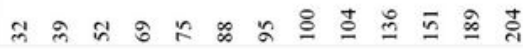

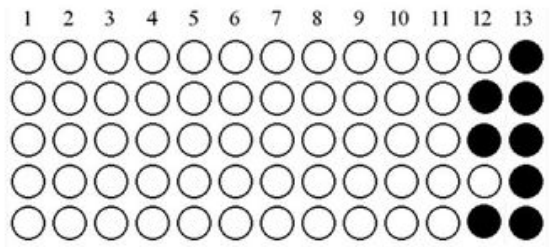

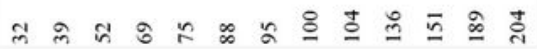

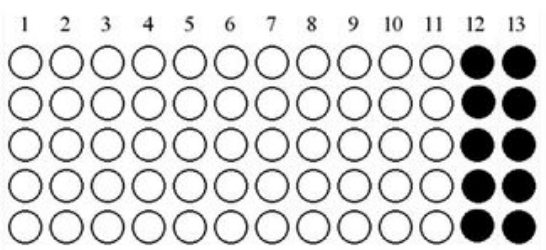

성

B

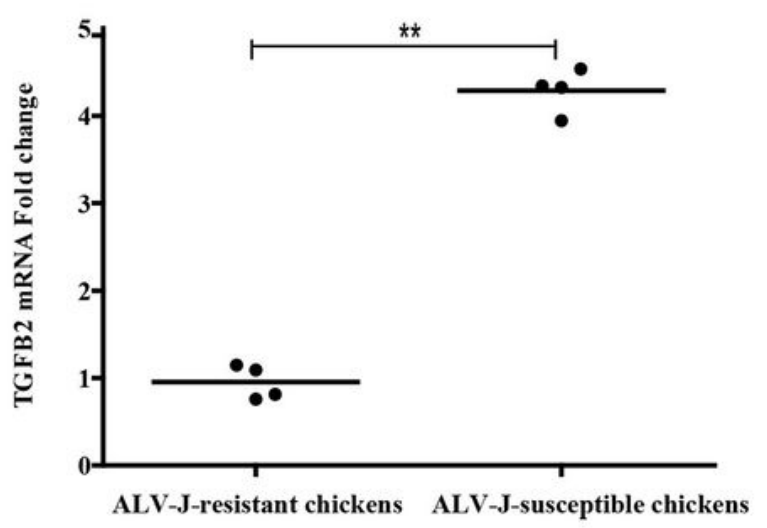

Figure 8

Identification of TGFB2 methylation level and expression in randomly selected chickens by BSP and qRTPCR. (A) The methylation status of TGFB2 in promoter region was investigated among randomly selected chickens by BSP. (B) Relative expression of TGFB2 in randomly selected chickens. Data shown are the means $\pm S E, * P<0.05$ and $* * P<0.01$. 

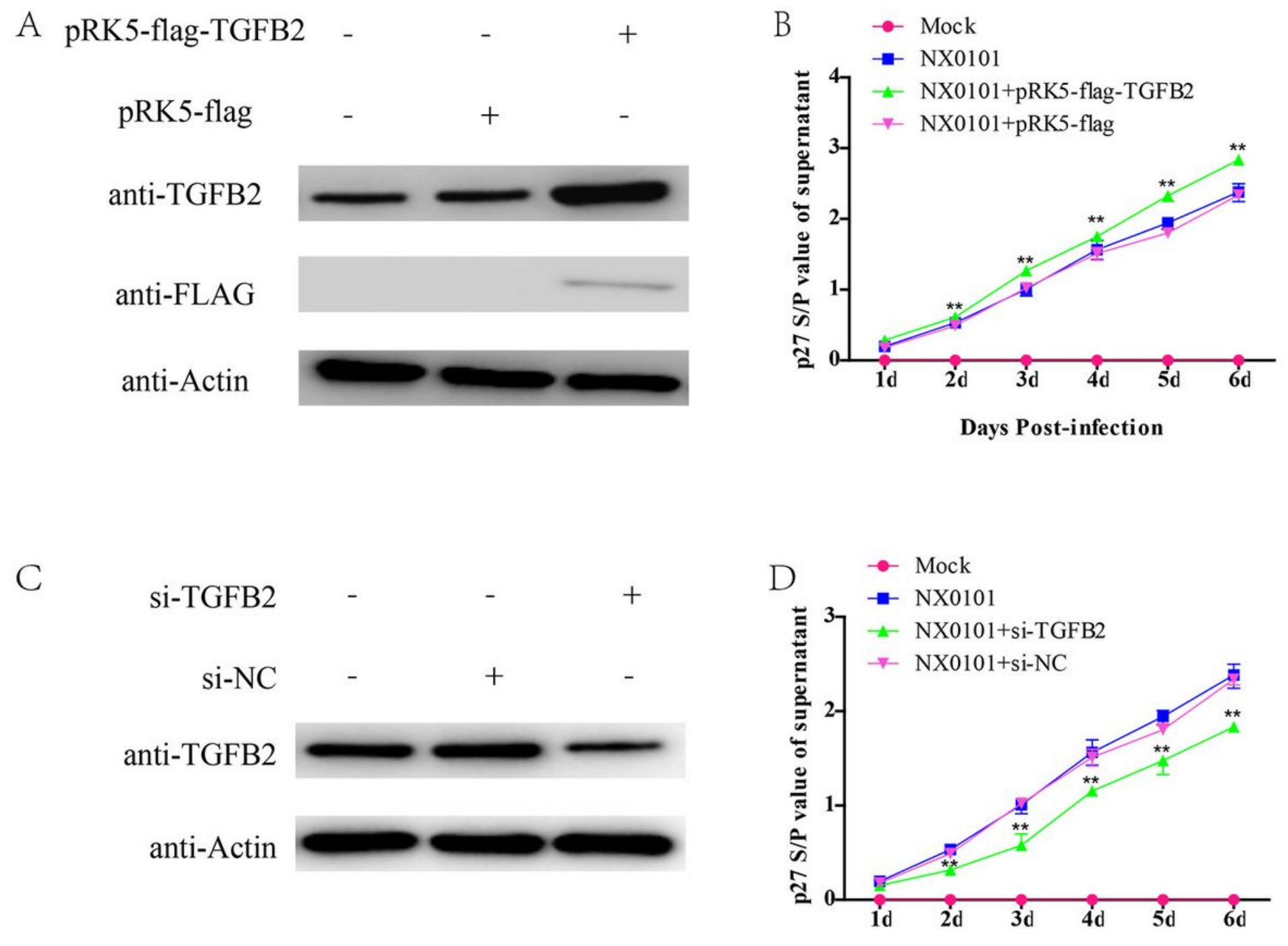

Days Post-infection

Figure 9

Effects of TGFB2 on ALV-J replication. (A) Western blotting of the proteins isolated $24 \mathrm{~h}$ after transfection of DF-1 cells with pRK5-flag-TGFB2 or pRK5-flag, and for the normal cells group. (B) Proliferation curve of ALV-J in DF-1 cells transfected with pRK5-flag-TGFB2 or pRK5-flag and challenged with ALV-J. (C) Western blotting of the proteins isolated $24 \mathrm{~h}$ after transfection of DF-1 cells with siRNA targeted to TGFB2 or si-NC, and for the normal cells group. (D) Proliferation curve of ALV-J in DF-1 cells transfected with the siRNA for TGFB2 or si-NC and challenged with ALV-J; ${ }^{\star} \mathrm{P}<0.05,{ }^{\star *} \mathrm{P}<0.01$.

\section{Supplementary Files}

This is a list of supplementary files associated with this preprint. Click to download.

- FigureS1.tif

- Tables1.xls 
- TableS10.xls

- Tables2.xls

- Tables3.xls

- Tables4.xls

- Tables5.xls

- Tables6.xIs

- Tables7.xls

- Tables8.xls

- Tables9.xls 